\title{
NORMAL APPROXIMATION OF U-STATISTICS IN HILBERT SPACE*
}

\author{
YU. V. BOROVSKIKH ${ }^{\dagger}$, L. MADAN PURI ${ }^{\ddagger}$, AND V. V. SAZONOV
}

(Translated by V. V. Sazonov)

\begin{abstract}
Let $\left\{U_{n}\right\}, n=1,2, \ldots$, be Hilbert space $H$-valued $U$-statistics with kernel $\Phi(\cdot, \cdot)$, corresponding to a sequence of observations (random variables) $X_{1}, X_{2}, \ldots$. The rate of convergence on balls in the central limit theorem for $\left\{U_{n}\right\}$ is investigated. The obtained estimate is of order $n^{-1 / 2}$ and depends explicitly on $\mathbf{E}\left\|\Phi\left(X_{1}, X_{2}\right)\right\|^{3}$ and on the trace and the first nine eigenvalues of the covariance operator of $\mathbf{E}\left(\Phi\left(X_{1}, X_{2}\right) \mid X_{1}\right)$.
\end{abstract}

Key words. $U$-statistic, Hilbert space, central limit theorem, normal (Gaussian) approximation, rate of convergence

\section{PII. S0040585X97975198}

1. Introduction. Let $X_{1}, \ldots, X_{n}$ be independent random variables with values in a measurable space $(X, \mathcal{X})$ having on it the same distribution $\mathbf{P}$. Let $H$ be a real separable Hilbert space with inner product $(\cdot, \cdot)$ and norm $\|\cdot\|$. Consider the $U H$-statistic

$$
U_{n}=\left(\begin{array}{l}
n \\
2
\end{array}\right)^{-1} \sum_{1 \leqq i<j \leqq n} \Phi\left(X_{i}, X_{j}\right)
$$

with a symmetric kernel $\Phi: X^{2} \rightarrow H$ such that $\mathbf{E} \Phi\left(X_{1}, X_{2}\right)=0, \mathbf{E}\left\|\Phi\left(X_{1}, X_{2}\right)\right\|<\infty$.

By the Hoeffding decomposition,

$$
U_{n}=2 n^{-1} \sum_{j=1}^{n} g_{1}\left(X_{j}\right)+2 n^{-1}(n-1)^{-1} \sum_{1 \leqq i<j \leqq n} g_{2}\left(X_{i}, X_{j}\right),
$$

where

$$
\begin{aligned}
g_{1}(x) & =\int_{X} \Phi(x, y) \mathbf{P}(d y)=\mathbf{E}\left(\Phi\left(X_{1}, X_{2}\right) \mid X_{1}=x\right), \\
g_{2}(x, y) & =\Phi(x, y)-g_{1}(x)-g_{1}(y) .
\end{aligned}
$$

We assume that $\sigma^{2}=\mathbf{E}\left\|g_{1}\left(X_{1}\right)\right\|^{2}>0$ and observe that the definitions of $g_{1}(x)$ and $g_{2}(x)$ imply

$$
\int_{X} g_{2}(x, y) \mathbf{P}(d y)=0 \quad(\text { a.s. })
$$

*Received by the editors May 17, 1994. This research was supported by the Office of Naval Research Contract N 00014-91-J-1020. The results of this paper were partially obtained during the visit of the last author to the Department of Management, Hong Kong University of Science and Technology, winter 1993-1994.

http://www.siam.org/journals/tvp/41-3/97519.html

†St.-Petersburg Transport University, Moskovskii pr. 9, 190031 St.-Peterburg, Russia.

‡Indiana University, Department of Mathematics, Bloomington, Indiana 47405, U.S.A.

ISteklov Mathematical Institute, Vavilov St. 42, 117966 Moscow, Russia. 
Assuming also that $\sigma<\infty$ we can define the covariance operator $V$ of $g_{1}\left(X_{1}\right)$ in the usual way:

$$
(V x, y)=\int_{X}\left(x, g_{1}(z)\right)\left(y, g_{1}(z)\right) \mathbf{P}(d z), \quad x, y \in H .
$$

Let $Y$ be a Gaussian $H$-valued random variable with mean zero and covariance operator $\sigma^{-2} V$. Denote by $\sigma_{1}^{2} \geqq \sigma_{2}^{2} \geqq \cdots$ the eigenvalues of $V$. Finally, for any $r \geqq 0$, $a \in H$, let $B_{r}(a)=\{x \in H:\|x-a\|<r\}$ and $B_{r}=B_{r}(0)$.

By the central limit theorem for $U H$-statistics, if

$$
\mathbf{E}\left\|g_{1}\left(X_{1}\right)\right\|^{2}<\infty, \quad \mathbf{E}\left\|g_{2}\left(X_{1}, X_{2}\right)\right\|^{4 / 3}<\infty
$$

then (see [1], [2], [5])

$$
\Delta_{n}(a)=\sup _{r}\left|\mathbf{P}\left\{\left\|2^{-1} n^{1 / 2} \sigma^{-1} U_{n}-a\right\|<r\right\}-\mathbf{P}\{\|Y-a\|<r\}\right| \longrightarrow 0, \quad n \rightarrow \infty .
$$

The rate of convergence of $\Delta_{n}(0)$ to zero with respect to $n$ was estimated in [1], [2], [5], [12], where under the additional moment conditions

$$
\mathbf{E}\left\|g_{1}\left(X_{1}\right)\right\|^{3}<\infty, \quad \mathbf{E}\left\|g_{2}\left(X_{1}, X_{2}\right)\right\|^{2}<\infty
$$

it was shown that

$$
\Delta_{n}(0)=\rho_{n}(V) n^{-1 / 2}
$$

with $\rho_{n}(V) \rightarrow 0$ as $n \rightarrow \infty$. To prove this, it was observed that the problem of estimating the probability for a value of the $U H$-statistic to be in the ball $B_{r}$ may be treated as the problem of estimating the rate of convergence in the central limit theorem in Hilbert space for balls with a nonzero random center which tends to zero with probability 1 as $n \rightarrow \infty$. However, it was assumed in [1], [2], [5], [12] that the number of nonzero eigenvalues of the covariance operator $V$ is sufficiently large. The explicit dependence of $\rho_{n}(V)$ on $V$ in [1], [2], [5], [12] was not determined. Estimates of order $O\left(n^{-1 / 3}\right)$ for any $a \in H$ with explicit dependence on the second and third moments were obtained in [14].

Note that in the one-dimensional case the study of the problem considered in the present paper has a rather long history. It was initiated in 1973 by Grams and Serfling [11] and later a number of gradual improvements of their result was made (for details see [13] and [10]). In [10] it was also shown that the estimate obtained by Korolyuk and Borovskikh in [4] is essentially unimprovable.

In the present paper we study the dependence of $\Delta_{n}(a)$ on $n$, the covariance operator $V$ and $\beta=\mathbf{E}\left\|\Phi\left(X_{1}, X_{2}\right)\right\|^{3}$. When $\beta<\infty$ an estimate of order $n^{-1 / 2}$ is obtained, the dependence of which on $\Phi$ involves only $\beta$, the first nine eigenvalues of $V$ and $\sigma^{2}=\operatorname{tr} V$. The order of dependence of our estimate on $n$ is precise, but its dependence on $\sigma_{j}^{2}, \sigma_{j}^{2}$ and $\beta$ apparently admits improvement. To prove the estimate we employ the methods developed in [3] and [12].

\section{The theorem.}

THEOREM. There exists an absolute constant $c$ such that, for all $n \geqq 2$ and $a \in H$,

$$
\left.\Delta_{n}(a) \leqq c\left(1+\|a\|^{3}\right) \sigma^{4} \quad \prod_{j=1}^{9} \sigma_{j}^{-1}\right) \sigma_{9}^{-1} \beta^{2} n^{-1 / 2} .
$$


In what follows we will assume that $\sigma=1$. The general case is reduced to this one if we replace $\Phi$ by $\sigma^{-1} \Phi$. We can assume also that $\beta<\infty$ and $\sigma_{9}>0$ since otherwise (3) would be obvious.

In the proof of the theorem the upper estimates for $\Delta_{n}(a)$ are calculated more precisely; except for $n$ and $V$, they depend only on the moments

$$
\mathbf{E}\left\|g_{1}\right\|^{3}=\mathbf{E}\left\|g_{1}\left(X_{1}\right)\right\|^{3}, \quad \mathbf{E}\left\|g_{2}\right\|^{3}=\mathbf{E}\left\|g_{2}\left(X_{1}, X_{2}\right)\right\|^{3},
$$

which are related to $\beta$ by the inequalities

$$
\mathbf{E}\left\|g_{1}\right\|^{3} \leqq \beta, \quad \mathbf{E}\left\|g_{2}\right\|^{3} \leqq 27 \beta .
$$

We formulate the theorem using only $\beta$ for the sake of simplicity.

3. Proof of the theorem. Below $c, c_{1}, c_{2}, \ldots$ denote absolute constants; the same symbol may stand for different constants. The proof is divided into several lemmas.

Lemma 1. We have

$$
\Delta_{n}(a) \leqq \Delta_{n 1}(a)+n \mathbf{P}\left\{\left\|g_{1}\left(X_{1}\right)\right\|>n^{1 / 2}\right\},
$$

where

$$
\begin{aligned}
\Delta_{n 1}(a) & =\sup _{r}\left|\mathbf{P}\left\{\left\|S_{n}-a+V_{n}\right\|<r\right\}-\mathbf{P}\{\|Y-a\|<r\}\right|, \\
S_{n} & =n^{-1 / 2} \sum_{j=1}^{n} g_{1}\left(X_{j}\right) I\left(\left\|g_{1}\left(X_{j}\right)\right\| \leqq n^{1 / 2}\right)
\end{aligned}
$$

$I(A)$ is the indicator function of the set $A$ and

$$
V_{n}=(n-1)^{-1} n^{-1 / 2} \sum_{j=1}^{n} \sum_{i=1}^{j-1} g_{2}\left(X_{i}, X_{j}\right) .
$$

Proof. According to (1),

$$
2^{-1} n^{1 / 2} U_{n}-a=S_{n}-a+V_{n}
$$

where

$$
S_{n}=n^{-1 / 2} \sum_{j=1}^{n} g_{1}\left(X_{j}\right)
$$

and we obviously have

$$
\Delta_{n}(a) \leqq \Delta_{n 1}(a)+\sup _{r}\left|\mathbf{P}\left\{\left\|S_{n}-a+V_{n}\right\|<r\right\}-\mathbf{P}\left\{\left\|S_{n}-a+V_{n}\right\|<r\right\}\right| .
$$

Now applying Lemma 1 of [17] with $f(x)=I(\|x\|<r), u_{1}=\infty, u_{2}=n^{1 / 2}$ and $Z=-a+V_{n}$, we see that the second summand in the right-hand side of this inequality is not greater than $n \mathbf{P}\left\{\left\|g_{1}\left(X_{1}\right)\right\|>n^{1 / 2}\right\}$ and the lemma follows. 
Let $k=n-\left[n^{2 / 3}\right]$, where $\left[n^{2 / 3}\right]$ is the integral part of $n^{2 / 3}$ and denote

$$
\begin{aligned}
\alpha_{k} & =\sum_{1 \leqq i<j \leqq k} g_{2}\left(X_{i}, X_{j}\right), \quad \beta_{k}=\sum_{i=1}^{k} \sum_{j=k+1}^{n} g_{2}\left(X_{i}, X_{j}\right), \\
\delta_{k} & =\sum_{k+1 \leqq i<j \leqq n} g_{2}\left(X_{i}, X_{j}\right), \quad S_{n}^{k}=n^{-1 / 2} \sum_{j=1}^{k} g_{1}\left(X_{j}\right), \\
g_{1}\left(X_{j}\right) & =g_{1}\left(X_{j}\right) I\left(\left\|g_{1}\left(X_{j}\right)\right\| \leqq n^{1 / 2}\right), \quad j=1, \ldots, n, \\
Z_{n} & =2 \varepsilon_{n}\left(S_{n}^{k}-a, \alpha_{k}\right), \quad \varepsilon_{n}=(n-1)^{-1} n^{-1 / 2} .
\end{aligned}
$$

Lemma 2. For $\Delta_{n 1}(a)$ we have

$$
\Delta_{n 1}(a) \leqq \Delta_{n 2}(a)+c_{1}\left(1+\|a\|^{3}\right)\left(\beta^{2}+\sigma_{1}^{-1} \sigma_{2}^{-1}\right) n^{-1 / 2},
$$

where

$$
\Delta_{n 2}(a)=\sup _{r}\left|\mathbf{P}\left\{\left\|S_{n}-a\right\|^{2}+Z_{n}<r\right\}-\mathbf{P}\left\{\|Y-a\|^{2}<r\right\}\right| .
$$

Proof. First we observe that

$$
\begin{aligned}
\left\|S_{n}-a+V_{n}\right\|^{2} & =\left(S_{n}-a+V_{n}, S_{n}-a+V_{n}\right) \\
& =\left\|S_{n}-a\right\|^{2}+2\left(S_{n}-a, V_{n}\right)+\left\|V_{n}\right\|^{2} .
\end{aligned}
$$

In our notation,

$$
\begin{gathered}
\left\|V_{n}\right\|^{2}=(n-1)^{-2} n^{-1}\left\|\sum_{j=2}^{n} \sum_{i=1}^{j-1} g_{2}\left(X_{i}, X_{j}\right)\right\|^{2}, \\
2\left(S_{n}-a, V_{n}\right)=Z_{n}+2 \varepsilon_{n}\left(S_{n}^{k}-a, \beta_{k}+\delta_{k}\right)+2\left(S_{n}-S_{n}^{k}, V_{n}\right),
\end{gathered}
$$

and

$$
\begin{aligned}
\left(S_{n}^{k}-a, \beta_{k}+\delta_{k}\right) & =\left(S_{n}^{k}, \beta_{k}\right)-\left(a, \beta_{k}\right)+\left(S_{n}^{k}, \delta_{k}\right)-\left(a, \delta_{k}\right), \\
\left(S_{n}-S_{n}^{k}, V_{n}\right) & =\varepsilon_{n}\left(S_{n}-S_{n}^{k}, \alpha_{k}\right)+\varepsilon_{n}\left(S_{n}-S_{n}^{k}, \beta_{k}\right)+\varepsilon_{n}\left(S_{n}-S_{n}^{k}, \delta_{k}\right) .
\end{aligned}
$$

In what follows we will need the following inequalities: for any $p \geqq 2$,

$$
\mathbf{E}\left\|S_{n}^{k}\right\|^{p} \leqq c_{p}, \quad\left\|\mathbf{E} g_{1}\left(X_{1}\right)\right\|=\left\|\int_{\left\|g_{1}(x)\right\|>n^{1 / 2}} g_{1}(x) \mathbf{P}(d x)\right\| \leqq n^{-1 / 2}
$$

(see [9], [16]) and

$$
\begin{aligned}
& \mathbf{E}\left\|\alpha_{k}\right\|^{p} \leqq c_{p} \mathbf{E}\left\|g_{2}\right\|^{p} k^{p}, \\
& \mathbf{E}\left\|\beta_{k}\right\|^{p} \leqq c_{p} \mathbf{E}\left\|g_{2}\right\|^{p} k^{p / 2}(n-k)^{p / 2}, \\
& \mathbf{E}\left\|\delta_{k}\right\|^{p} \leqq c_{p} \mathbf{E}\left\|g_{2}\right\|^{p}(n-k)^{p}
\end{aligned}
$$

(see [5]).

The Chebychev inequality and (9) imply

$$
\mathbf{P}\left\{\left\|V_{n}\right\|^{2} \geqq n^{-1 / 2}\right\} \leqq n^{1 / 2} \mathbf{E}\left\|V_{n}\right\|^{2} \leqq c_{1} \mathbf{E}\left\|g_{2}\right\|^{2} n^{-1 / 2} .
$$


Furthermore,

$$
\mathbf{P}\left\{\left|\left(S_{n}^{k}, \varepsilon_{n} \beta_{k}\right)\right|>n^{-1 / 2}\right\} \leqq \Pi_{1}+\Pi_{2},
$$

where

$$
\begin{gathered}
\left.\Pi_{1}=\mathbf{P}\left\{2 k \varepsilon_{n} \mid \mathbf{E} g_{1}\left(X_{1}\right), \sum_{i=1}^{k} h\left(X_{i}\right)\right) \mid>1\right\}, \\
\left.\Pi_{2}=\mathbf{P}\left\{2 \varepsilon_{n} \mid \sum_{i=1}^{k} g\left(X_{i}\right), \sum_{i=1}^{k} h\left(X_{i}\right)\right) \mid>1\right\}, \\
g\left(X_{i}\right)=g_{1}\left(X_{i}\right)-\mathbf{E} g_{1}\left(X_{1}\right), \quad h\left(X_{i}\right)=\sum_{j=k+1}^{n} g_{2}\left(X_{i}, X_{j}\right), \quad i=1, \ldots, k .
\end{gathered}
$$

For $\Pi_{1}$ by the Chebychev inequality and (8), (9)

$$
\Pi_{1} \leqq\left(2 k \varepsilon_{n}\right)^{3}\left\|\mathbf{E} g_{1}\left(X_{1}\right)\right\|^{3} \mathbf{E}\left\|\sum_{i=1}^{k} h\left(X_{i}\right)\right\|^{3} \leqq c_{1} \mathbf{E}\left\|g_{2}\right\|^{3} n^{-1 / 2} .
$$

To estimate $\Pi_{2}$ we first observe that

$$
\left.\sum_{i=1}^{k} g\left(X_{i}\right), \sum_{i=1}^{k} h\left(X_{i}\right)\right)=\sum_{i=1}^{4} A_{i}
$$

where

$$
\begin{aligned}
& A_{1}=k \mathbf{E}_{1}\left(g\left(X_{1}\right), h\left(X_{1}\right)\right) \\
& A_{2}=\sum_{i=1}^{k}\left(\left(g\left(X_{i}\right), h\left(X_{i}\right)\right)-\mathbf{E}_{1}\left(g\left(X_{1}\right), h\left(X_{1}\right)\right)\right) \\
& A_{3}=\sum_{i_{1}=2}^{k} \sum_{i_{2}=1}^{i_{1}-1}\left(g\left(X_{i_{1}}\right), h\left(X_{i_{2}}\right)\right), \quad A_{4}=\sum_{i_{1}=1}^{k-1} \sum_{i_{2}=i_{1}+1}^{k}\left(g\left(X_{i_{1}}\right), h\left(X_{i_{2}}\right)\right)
\end{aligned}
$$

and, obviously,

$$
\mathbf{E}_{k}\left|A_{1}\right|^{3} \leqq k^{3}\left|\mathbf{E}_{1}\left(g\left(X_{1}\right), h\left(X_{1}\right)\right)\right|^{3}
$$

by $[8]$

$$
\mathbf{E}_{k}\left|A_{2}\right|^{3} \leqq c_{1} k^{3 / 2} \mathbf{E}_{1}\left|\left(g\left(X_{1}\right), h\left(X_{1}\right)\right)\right|^{3}
$$

and by $[9]$

$$
\mathbf{E}_{k}\left|A_{3}\right|^{3} \leqq c_{1} k^{3} \mathbf{E}_{2}\left|\left(g\left(X_{1}\right), h\left(X_{2}\right)\right)\right|^{3}, \quad \mathbf{E}_{k}\left|A_{4}\right|^{3} \leqq c_{1} k^{3} \mathbf{E}_{2}\left|\left(g\left(X_{1}\right), h\left(X_{2}\right)\right)\right|^{3},
$$

$\mathbf{E}_{k}(\cdot)$ denotes the expectation with respect to $X_{1}, \ldots, X_{k}$ with fixed $X_{k+1}, \ldots, X_{n}$, $k=1, \ldots, n$.

Moreover, since $\mathbf{E}\left\|g_{1}\left(X_{1}\right)\right\|^{2}=1$, we have

$$
\left|\mathbf{E}\left(g\left(X_{1}\right), h\left(X_{1}\right)\right)\right|^{3} \leqq \mathbf{E}_{1}\left\|h\left(X_{1}\right)\right\|^{3} .
$$


And, obviously,

$$
\begin{aligned}
& \mathbf{E}_{1}\left|\left(g\left(X_{1}\right), h\left(X_{1}\right)\right)\right|^{3} \leqq 8 n^{3 / 2} \mathbf{E}_{1}\left\|h\left(X_{1}\right)\right\|^{3} \\
& \mathbf{E}_{2}\left|\left(g\left(X_{1}\right), h\left(X_{2}\right)\right)\right|^{3} \leqq c_{1} \mathbf{E}_{1}\left\|g\left(X_{1}\right)\right\|^{3} \mathbf{E}_{1}\left\|h\left(X_{1}\right)\right\|^{3}
\end{aligned}
$$

and by $[8]$

$$
\mathbf{E}_{k}\left\|h\left(X_{1}\right)\right\|^{3} \leqq c_{1}(n-k)^{3 / 2} \mathbf{E}_{k}\left\|g_{2}\left(X_{1}, X_{k+1}\right)\right\|^{3},
$$

$\mathbf{E}_{k}(\cdot)$ denotes the expectation with respect to $X_{k+1}, \ldots, X_{n}$ with fixed $X_{1}, \ldots, X_{k}$, $k=1, \ldots, n$.

The above implies

$$
\Pi_{2} \leqq c_{1} \mathbf{E}\left\|g_{1}\right\|^{3} \mathbf{E}\left\|g_{2}\right\|^{3} n^{-1 / 2}
$$

and, hence we obtain an estimate for the probability in the left side of (10).

Similarly, using (8), (9) we get

$$
\begin{aligned}
& \mathbf{P}\left\{\left|c\left(S_{n}^{k}, \varepsilon_{n} \delta_{k}\right)\right|>n^{-1 / 2}\right\} \leqq \mathbf{P}\left\{c \|\left(S_{n}^{k} \|>n^{1 / 6}\right\}+\mathbf{P}\left\{n^{1 / 6} \varepsilon_{n}\left\|\delta_{k}\right\|>n^{-1 / 2}\right\}\right. \\
& \leqq c^{3} \mathbf{E}\left\|S_{n}^{k}\right\|^{3} n^{-1 / 2}+n^{1 / 2} n^{3 / 2} \varepsilon_{n}^{3} \mathbf{E}\left\|\delta_{k}\right\|^{3} \leqq c_{1} n^{-1 / 2}+c_{2} \mathbf{E}\left\|g_{2}\right\|^{3} n^{-1 / 2}, \\
& \mathbf{P}\left\{\left|c\left(a, \varepsilon_{n} \beta_{k}\right)\right|>n^{-1 / 2}\right\} \leqq c^{3}\|a\|^{3} \varepsilon_{n}^{3} n^{3 / 2} \mathbf{E}\left\|\beta_{k}\right\|^{3} \leqq c_{1} \mathbf{E}\|a\|^{3} \mathbf{E}\left\|g_{2}\right\|^{3} n^{-1 / 2}, \\
& \mathbf{P}\left\{\left|c\left(a, \varepsilon_{n} \delta_{k}\right)\right|>n^{-1 / 2}\right\} \leqq c^{3}\|a\|^{3} \varepsilon_{n}^{3} n^{3 / 2} \mathbf{E}\left\|\delta_{k}\right\|^{3} \leqq c_{1} \mathbf{E}\|a\|^{3} \mathbf{E}\left\|g_{2}\right\|^{3} n^{-1 / 2}, \\
& \mathbf{P}\left\{\left|c\left(S_{n}-S_{n}^{k}, \varepsilon_{n} \alpha_{k}\right)\right|>n^{-1 / 2}\right\} \leqq c^{3} n^{3 / 2} \mathbf{E}\left\|S_{n}-S_{n}^{k}\right\|^{3} \varepsilon_{n}^{3} \mathbf{E}\left\|\alpha_{k}\right\|^{3} \\
& \quad \leqq c_{1} \mathbf{E}\left\|g_{1}\right\|^{3} \mathbf{E}\left\|g_{2}\right\|^{3} n^{-1 / 2}, \\
& \mathbf{P}\left\{c\left|\left(S_{n}-S_{n}^{k}, \varepsilon_{n} \beta_{k}\right)\right|>n^{-1 / 2}\right\} \leqq \mathbf{P}\left\{c\left\|S_{n}-S_{n}^{k}\right\|>1\right\}+\mathbf{P}\left\{\varepsilon_{n}\left\|\beta_{k}\right\|>n^{-1 / 2}\right\} \\
& \quad \leqq c^{3} \mathbf{E}\left\|S_{n}-S_{n}^{k}\right\|^{3}+n^{3 / 2} \varepsilon_{n}^{3} \mathbf{E}\left\|\beta_{k}\right\|^{3} \leqq c_{1} \mathbf{E}\left\|g_{1}\right\|^{3} n^{-1 / 2}+c_{2} \mathbf{E}\left\|g_{2}\right\|^{3} n^{-1 / 2}, \\
& \mathbf{P}\left\{\left|c\left(S_{n}-S_{n}^{k}, \varepsilon_{n} \delta_{k}\right)\right|>n^{-1 / 2}\right\} \leqq \mathbf{P}\left\{c\left\|S_{n}-S_{n}^{k}\right\|>1\right\}+\mathbf{P}\left\{\varepsilon_{n}\left\|\delta_{k}\right\|>n^{-1 / 2}\right\} \\
& \quad \leqq c^{3} \mathbf{E}\left\|S_{n}-S_{n}^{k}\right\|^{3}+n^{3 / 2} \varepsilon_{n}^{3} \mathbf{E}\left\|\delta_{k}\right\|^{3} \leqq c_{1} \mathbf{E}\left\|g_{1}\right\|^{3} n^{-1 / 2}+c_{2} \mathbf{E}\left\|g_{2}\right\|^{3} n^{-1 / 2} .
\end{aligned}
$$

Denote now

$$
Y_{n}=2 \varepsilon_{n}\left(S_{n}^{k}-a, \beta_{k}+\delta_{k}\right)+2\left(S_{n}-S_{n}^{k}, V_{n}\right)+\left\|V_{n}\right\|^{2} .
$$

Then, according to (7),

$$
\left\|S_{n}-a+V_{n}\right\|^{2}=\left\|S_{n}-a\right\|^{2}+Z_{n}+Y_{n}
$$

and the estimates above imply

$$
\mathbf{P}\left\{\left|Y_{n}\right|>n^{-1 / 2}\right\} \leqq c_{1}\left(1+\|a\|^{3}\right) \mathbf{E}\left\|g_{1}\right\|^{3} \mathbf{E}\left\|g_{2}\right\|^{3} n^{-1 / 2} .
$$

Since, for all $r \geqq 0$,

$$
\begin{aligned}
& \mathbf{P}\left\{\left\|S_{n}-a\right\|^{2}+Z_{n}<r-n^{-1 / 2}\right\}-\mathbf{P}\left\{\left|Y_{n}\right|>n^{-1 / 2}\right\} \\
& \quad \leqq \mathbf{P}\left\{\left\|S_{n}-a\right\|^{2}+Z_{n}+Y_{n}<r\right\} \\
& \quad \leqq \mathbf{P}\left\{\left\|S_{n}-a\right\|^{2}+Z_{n}<r+n^{-1 / 2}\right\}+\mathbf{P}\left\{\left|Y_{n}\right|>n^{-1 / 2}\right\},
\end{aligned}
$$


we have

$$
\Delta_{n 1}(a) \leqq \Delta_{n 2}(a)+\mathbf{P}\left\{\left|Y_{n}\right|>n^{-1 / 2}\right\}+\sup _{r} \mathbf{P}\left\{r-n^{-1 / 2} \leqq\|Y-a\|^{2} \leqq r+n^{-1 / 2}\right\} .
$$

In $[6]$ it is proved that

$$
\sup _{r} \mathbf{P}\left\{r-n^{-1 / 2} \leqq\|Y-a\|^{2} \leqq r+n^{-1 / 2}\right\} \leqq c \sigma_{1}^{-1} \sigma_{2}^{-1} n^{-1 / 2} .
$$

Estimates (11)-(13) imply (6).

Denote now

$$
\begin{aligned}
\varphi_{n}(t) & =\mathbf{E} \exp \left(i t\left\|S_{n}-a\right\|^{2}+i t Z_{n}\right), \\
\varphi(t) & =\mathbf{E} \exp \left(i t\|Y-a\|^{2}\right), \\
\psi_{n}(t) & =\mathbf{E} \exp \left(i t\left\|S_{n}-a\right\|^{2}\right) .
\end{aligned}
$$

The density of the random variable $\|Y-a\|^{2}$ is not greater than $c\left(\sigma_{1} \sigma_{2}\right)^{-1}$ (see [6]). Thus for $\Delta_{n 2}(a)$ in (6), applying Esséen's inequality (see, e.g. [7]) for any $T>0$ we have

$$
\Delta_{n 2}(a) \leqq c_{1} \int_{-T}^{T}\left|\varphi_{n}(t)-\varphi(t)\right||t|^{-1} d t+c_{2}\left(\sigma_{1} \sigma_{2}\right)^{-1} T^{-1} .
$$

We choose in (14)

$$
T=\frac{c n^{1 / 2}}{L}, \quad L=3 \sigma_{9}^{-2} \mathbf{E}\left\|g_{1}\right\|^{3}, \sigma_{9}>0,
$$

where $c$ is a small enough absolute constant and then apply Lemma 12 of [3]. Note that with $L$ as in (15) the following conditions of Lemma 12 in [3]

$$
\frac{2}{3} \leqq L^{2} \leqq \frac{n}{2 e}, \quad \mathbf{P}\left(B_{L}\right) \geqq \frac{1}{2}
$$

are fulfilled (for a proof see [3], [9], [16]). When (16) is satisfied the distribution of $g_{1}=g_{1}\left(X_{1}\right) I\left(\left\|g_{1}\left(X_{1}\right)\right\| \leqq n^{1 / 2}\right)$ may be represented as $\left(Q+Q_{1}\right) / 2$, where

$$
Q(A)=\frac{\mathbf{P}\left(A \cap B_{L}\right)}{\mathbf{P}\left(B_{L}\right)}, \quad A \in H,
$$

and $Q_{1}$ is a probability measure (see [15]).

Let $V_{1}$ be the covariance operator of $Q$ and $\sigma_{11}^{2} \geqq \sigma_{12}^{2} \geqq \cdots$ be its eigenvalues. By our assumptions, $\operatorname{tr} V=1$. Hence (see [3]) $\operatorname{tr} V_{1} \leqq 2$ and

$$
\frac{2 \sigma_{j}^{2}}{3} \leqq \sigma_{1 j}^{2} \leqq 2 \sigma_{j}^{2}, \quad j=1, \ldots, 9 .
$$

To estimate the integral in (14) we will need the following generalization of Lemma 11 in [3] (see also [9, Lemma 4]).

Lemma 3. Denote

$$
Z=n^{-1 / 2} \sum_{j=1}^{l+m} g_{1}\left(X_{j}\right), \quad Z_{1}(f)=\sum_{j=1}^{l+m} f\left(X_{j}\right),
$$


where $g_{1}=g_{1}\left(X_{1}\right) I\left(\left\|g_{1}\left(X_{1}\right)\right\| \leqq n^{1 / 2}\right)$ and $f: X \rightarrow \mathbf{R}$ is a measurable function, $l, m$ are positive integers, $l \leqq m, l+m \leqq n$. Then, for any $A>0$, even $k \geqq 0$, integer $r \geqq 0, k_{j} \geqq 0, j=1, \ldots, r$, and any $x, x_{j} \in H, j=1, \ldots, r$, for $|t| \leqq$ $c(A) n L^{-1}\left(l \log \left(l / L^{2}\right)\right)^{-1 / 2}$ if $l>L^{2}$, and for any $t$ if $l \leqq L^{2}$

$$
\begin{aligned}
& \left|\mathbf{E} \exp \left(i t\|Z+x\|^{2}+i t Z_{1}(F)\right)\|Z\|^{k} \prod_{j=1}^{r}\left(x_{j}, Z\right)^{k_{j}}\right| \\
& \left.\quad \leqq c_{1} \prod_{j=1}^{r}\left\|x_{j}\right\|^{k_{j}} \exp \left(-c_{2} l\right)+c_{3}\left(\begin{array}{c}
L^{2} \\
l
\end{array}\right)^{A}+h^{1 / 2}\left(\frac{c_{4} T_{1}^{2} l m}{n^{2}}, V_{1}\right)\right),
\end{aligned}
$$

where $c_{1}, c_{2}$ are functions of $k=k+k_{1}+\cdots+k_{r} ; c_{3}, c_{4}$ are functions of $A$ and $k$

$$
T_{1}=\min \left\{|t|, n L^{-1}\left(m \log \left(\frac{m}{L^{2}}\right)\right)^{-1 / 2}\right\}, \quad h\left(s, V_{1}\right)=\prod_{j=1}^{\infty}\left(1+2 s \sigma_{1 j}^{4}\right)^{-1 / 2} .
$$

Proof. This lemma reduces to Lemma 11 in [3] if $Z_{1}(f)=0$. The proof of the present lemma is essentially the same as the proof of Lemma 11 in [3].

Let us estimate the integral in (14). Obviously,

$$
\int_{-T}^{T}\left|\varphi_{n}(t)-\varphi(t)\right||t|^{-1} d t \leqq I_{1}+I_{2}
$$

where

$$
I_{1}=\int_{-T}^{T}\left|{ }_{n}(t)-\varphi(t)\right||t|^{-1} d t, \quad I_{2}=\int_{-T}^{T}\left|\varphi_{n}(t)-\psi_{n}(t)\right||t|^{-1} d t .
$$

By Lemma 12 in [3]

$$
I_{1} \leqq c_{1} U
$$

where

$$
\begin{aligned}
U= & c_{7}(V)\left(\mathbf{E}\left(\left\|g_{1}\right\|^{2}+\left(a, g_{1}\right)^{2}\right) I\left(\left\|g_{1}\right\|>n^{1 / 2}\right)\right) \\
& +\left(\mathbf{E}\left(\left\|g_{1}\right\|^{3}+\left|\left(a, g_{1}\right)\right|^{3}\right) I\left(\left\|g_{1}\right\| \leqq n^{1 / 2}\right)+(V a, a)^{3 / 2}\right) n^{-1 / 2} \\
& +c_{3}(V) \sigma_{9}^{-2} \mathbf{E}\left\|g_{1}\right\|^{3} n^{-1 / 2}, \quad c_{s}(V)=\prod_{j=1}^{s} \sigma_{j}^{-1},
\end{aligned}
$$

and for $U$ we have

$$
\begin{aligned}
U & \leqq\left(1+\|a\|^{3}\right) c_{7}(V) \mathbf{E}\left\|g_{1}\right\|^{3} n^{-1 / 2}+c_{3}(V) \sigma_{9}^{-2} \mathbf{E}\left\|g_{1}\right\|^{3} n^{-1 / 2} \\
& \leqq 2\left(1+\|a\|^{3}\right) \beta c_{9}(V) \sigma_{9}^{-1} n^{-1 / 2} .
\end{aligned}
$$

Consider now $I_{2}$ in (19). Obviously,

$$
\varphi_{n}(t)-\psi_{n}(t)=\mathbf{E} \exp \left\{i t\left\|S_{n}-a\right\|^{2}\right\}\left(e^{i t Z_{n}}-1\right) .
$$

Next we will use the representation

$$
e^{z}-1=\sum_{s=1}^{r-1} \frac{z^{s}}{s !}+z^{r} \int_{0}^{1} \frac{(1-u)^{r-1} e^{u z}}{(r-1) !} d u
$$


valid for all complex $z$ and positive integers $r$, with $r=3, z=i t Z_{n}$. Thus we have

$$
\varphi_{n}(t)-\psi_{n}(t)=i t J_{1}(t)-\frac{t^{2}}{2} J_{2}(t)+\frac{(i t)^{3}}{2} J_{3}(t),
$$

where

$$
\begin{aligned}
& J_{1}(t)=\mathbf{E}\left(Z_{n} \exp \left\{i t\left\|S_{n}-a\right\|^{2}\right\}\right), \quad J_{2}(t)=\mathbf{E}\left(Z_{n}^{2} \exp \left\{i t\left\|S_{n}-a\right\|^{2}\right\}\right), \\
& J_{3}(t)=\int_{0}^{1} d u(1-u)^{2} \mathbf{E}\left(Z_{n}^{3} \exp \left\{i t\left\|S_{n}-a\right\|^{2}+i t u Z_{n}\right\}\right) .
\end{aligned}
$$

Lemma 4. If $|t| \leqq T=c n^{1 / 2} L^{-1}$, then

$$
\begin{aligned}
\left|J_{3}(t)\right| \leqq & \left.c_{1} \quad L^{3 / 2} n^{-1 / 2}+\prod_{j=1}^{7}\left(1+\frac{c_{2} \sigma_{j}^{4} t^{2}}{n^{2 / 3}}\right)^{-1 / 4}\right) \\
& \times\left(\mathbf{E}\left\|g_{1}\right\|^{3}+\|a\|^{3}\right) \mathbf{E}\left\|g_{2}\right\|^{3} n^{-3 / 2} .
\end{aligned}
$$

Proof. The random variable $Z_{n}$ does not depend on $X_{k+1}, \ldots, X_{n}$. Hence,

$$
\left|\mathbf{E}\left(Z_{n}^{3} \exp \left\{i t\left\|S_{n}-a\right\|^{2}+i t u Z_{n}\right\}\right)\right| \leqq \mathbf{E}_{k}\left|Z_{n}\right|^{3}\left|\mathbf{E}_{k} \exp \left\{i t\left\|S_{n}-a\right\|^{2}\right\}\right|,
$$

where as above $\mathbf{E}_{k}(\cdot)$ denotes expectation with respect to $X_{k+1}, \ldots, X_{n}$ with fixed $X_{1}, \ldots, X_{k}$. Representing $S_{n}=\left(S_{n}-S_{n}^{k}\right)+S_{n}^{k}$ we apply Lemma 3 with $Z=S_{n}-S_{n}^{k}$, $1 \sim n^{2 / 3} / 4, m \sim 3 n^{2 / 3} / 4, A={ }_{4}^{3}$. Noting that here $T_{1}=|t|$, we obtain

$$
\begin{aligned}
& \left|\mathbf{E}_{k} \exp \left\{i t\left\|S_{n}-a\right\|^{2}\right\}\right| \\
& \left.\quad \leqq c_{1} \quad \exp \left(-c_{2} n^{2 / 3}\right)+c_{3} L^{3 / 2} n^{-1 / 2}+\prod_{j=1}^{7}\left(1+\frac{c_{4} \sigma_{j}^{4} t^{2}}{n^{2 / 3}}\right)^{-1 / 4}\right) .
\end{aligned}
$$

To estimate $\mathbf{E}\left|Z_{n}\right|^{3}$ we first write

$$
\mathbf{E}\left|Z_{n}\right|^{3} \leqq 32 \varepsilon_{n}^{3}\left(\mathbf{E}\left|\left(S_{n}^{k}, \alpha_{k}\right)\right|^{3}+\|a\|^{3} \mathbf{E}\left\|\alpha_{k}\right\|^{3}\right) .
$$

Then we represent the scalar product $\left(S_{n}^{k}, \alpha_{k}\right)$ in the form

$$
\begin{aligned}
\left(S_{n}^{k}, \alpha_{k}\right)= & \left.n^{-1 / 2} \sum_{j=1}^{k} g\left(X_{j}\right), \sum_{1 \leqq i_{1}<i_{2} \leqq k} g_{2}\left(X_{i_{1}}, X_{i_{2}}\right)\right) \\
& +n^{-1 / 2} k\left(\mathbf{E} g_{1}\left(X_{1}\right), \sum_{1 \leqq i_{1}<i_{2} \leqq k} g_{2}\left(X_{i_{1}}, X_{i_{2}}\right)\right) .
\end{aligned}
$$

Using (8)-(9) we have

$$
\begin{aligned}
\mathbf{E} \mid & \left.\mathbf{E} g_{1}\left(X_{1}\right), \sum_{1 \leqq i_{1}<i_{2} \leqq k} g_{2}\left(X_{i_{1}}, X_{i_{2}}\right)\right)\left.\right|^{3} \\
& \leqq\left\|\mathbf{E} g_{1}\left(X_{1}\right)\right\|^{3} \mathbf{E}\left\|\sum_{1 \leqq i_{1}<i_{2} \leqq k} g_{2}\left(X_{i_{1}}, X_{i_{2}}\right)\right\|^{3} \leqq c_{1} n^{-3 / 2} k^{3} \mathbf{E}\left\|g_{2}\right\|^{3} .
\end{aligned}
$$


To estimate the first term in the right side of (26) we will use the representation

$$
\begin{aligned}
& \left.\sum_{j=1}^{k} g_{1}\left(X_{j}\right), \sum_{1 \leqq i_{1}<i_{2} \leqq k} g_{2}\left(X_{i_{1}}, X_{i_{2}}\right)\right) \\
& =\sum_{j=1}^{k} \sum_{1 \leqq i_{1}<i_{2} \leqq k}\left(g_{1}\left(X_{j}\right), g_{2}\left(X_{i_{1}}, X_{i_{2}}\right)\right)=\sum_{i=1}^{5} G_{i},
\end{aligned}
$$

where

$$
\begin{aligned}
G_{1} & =\sum_{j=3}^{k} \sum_{i_{2}=2}^{j-1} \sum_{i_{1}=1}^{i_{2}-1}\left(g\left(X_{j}\right), g_{2}\left(X_{i_{1}}, X_{i_{2}}\right)\right), \\
G_{2} & =\sum_{j=2}^{k} \sum_{i_{1}=1}^{j-1}\left(g\left(X_{j}\right), g_{2}\left(X_{i_{1}}, X_{j}\right)\right), \\
G_{3} & =\sum_{j=2}^{k-1} \sum_{i_{1}=1}^{j-1} \sum_{i_{2}=j+1}^{k}\left(g\left(X_{j}\right), g_{2}\left(X_{i_{1}}, X_{i_{2}}\right)\right), \\
G_{4} & =\sum_{j=1}^{k} \sum_{i_{2}=j+1}^{k}\left(g\left(X_{j}\right), g_{2}\left(X_{j}, X_{i_{2}}\right)\right), \\
G_{5} & =\sum_{j=1}^{k} \sum_{i_{2}=j+2}^{k} \sum_{i_{1}=j+1}^{i_{2}-1}\left(g\left(X_{j}\right), g_{2}\left(X_{i_{1}}, X_{i_{2}}\right)\right) .
\end{aligned}
$$

By (9) we have

$$
\begin{array}{ll}
\mathbf{E}\left|G_{i}\right|^{3} \leqq c_{1} \mathbf{E}\left\|g_{1}\right\|^{3} \mathbf{E}\left\|g_{2}\right\|^{3} n^{9 / 2}, & i=1,3,5, \\
\mathbf{E}\left|G_{i}\right|^{3} \leqq c_{2} \mathbf{E}\left\|g_{2}\right\|^{3} n^{9 / 2}, & i=2,4 .
\end{array}
$$

Combining (26) and (27) we obtain

$$
\mathbf{E}\left|Z_{n}\right|^{3} \leqq c_{1}\left(\mathbf{E}\left\|g_{1}\right\|^{3}+\|a\|^{3}\right) \mathbf{E}\left\|g_{2}\right\|^{3} n^{-3 / 2} .
$$

Together with (24) this implies (23).

For $m \sim n$ partition the interval $|t| \leqq T=c n^{1 / 2} L^{-1}$ into the two subsets

$$
|t| \leqq T(m)=n L^{-1}\left(m \log \frac{m}{L^{2}}\right)^{-1 / 2}
$$

and

$$
T(m) \leqq|t| \leqq T .
$$

Lemma 5. For $t$ satisfying (28)

$$
\begin{aligned}
\left|J_{1}(t)\right| \leqq & c_{1}\left(1+\|a\|^{3}\right)\left(\mathbf{E}\left\|g_{1}\right\|^{3}\right)\left(\mathbf{E}\left\|g_{2}\right\|^{3}\right)^{1 / 3} \\
& \times \quad L^{3} n^{-1 / 2}+\left(|t|+t^{2}\right) \prod_{j=1}^{7}\left(1+c_{2} \sigma_{j}^{4} t^{2}\right)^{-1 / 4} \\
& \left.+|t|^{3} \prod_{j=1}^{9}\left(1+c_{2} \sigma_{j}^{4} t^{2}\right)^{-1 / 4}\right) n^{-1 / 2}
\end{aligned}
$$


and for $t$ satisfying (29)

$$
\left|J_{1}(t)\right| \leqq c_{1}\left(1+\|a\|^{3}\right)\left(\mathbf{E}\left\|g_{1}\right\|^{3}\right)\left(\mathbf{E}\left\|g_{2}\right\|^{3}\right)^{1 / 3} c_{9}(V) L^{2} n^{-1} .
$$

Proof. Let us show first that

$$
J_{1}(t)=4 n^{-1 / 2} \varepsilon_{n}\left(\begin{array}{l}
k \\
2
\end{array}\right) h_{1}(t)+2(k-2) n^{-1 / 2} \varepsilon_{n}\left(\begin{array}{l}
k \\
2
\end{array}\right) h_{2}(t)-2 \varepsilon_{n}\left(\begin{array}{l}
k \\
2
\end{array}\right) h_{3}(t),
$$

where

$$
\begin{aligned}
h_{1}(t)= & \mathbf{E}\left(\left(g_{1}\left(X_{1}\right), g_{2}\left(X_{1}, X_{2}\right)\right) \exp \left\{i t\left\|g_{1 n}\left(X_{1}\right)+g_{1 n}\left(X_{2}\right)+S_{n 2}\right\|^{2}\right\}\right), \\
h_{2}(t)=\mathbf{E}( & \left(g_{1}\left(X_{1}\right), g_{2}\left(X_{2}, X_{3}\right)\right) \\
& \left.\quad \times \exp \left\{i t\left\|g_{1 n}\left(X_{1}\right)+g_{1 n}\left(X_{2}\right)+g_{1 n}\left(X_{3}\right)+S_{n 3}\right\|^{2}\right\}\right), \\
h_{3}(t)= & \mathbf{E}\left(\left(a, g_{2}\left(X_{1}, X_{2}\right)\right) \exp \left\{i t\left\|g_{1 n}\left(X_{1}\right)+g_{1 n}\left(X_{2}\right)+S_{n 2}\right\|^{2}\right\}\right), \\
g_{1 n}\left(X_{j}\right)= & g_{1}\left(X_{j}\right) n^{-1 / 2}, \quad S_{n r}=n^{-1 / 2} \sum_{j=r+1}^{n} g_{1}\left(X_{j}\right)-a .
\end{aligned}
$$

Indeed, according to the definition,

$$
\begin{aligned}
J_{1}(t) & =2 n^{-1 / 2} \varepsilon_{n} \sum_{i=1}^{5} H_{i}(t)-2 \varepsilon_{n} H_{6}, \\
H_{1}(t) & =\sum_{j=3}^{k} \sum_{i_{2}=2}^{j-1} \sum_{i_{1}=1}^{i_{2}-1} \mathbf{E}\left(\left(g_{1}\left(X_{j}\right), g_{2}\left(X_{i_{1}}, X_{i_{2}}\right)\right) \exp \left\{i t\left\|S_{n}-a\right\|^{2}\right\}\right)=\left(\begin{array}{l}
k \\
3
\end{array}\right) h_{2}(t), \\
H_{2}(t) & =\sum_{j=2}^{k} \sum_{i_{2}=1}^{j-1} \mathbf{E}\left(\left(g_{1}\left(X_{j}\right), g_{2}\left(X_{i_{2}}, X_{j}\right)\right) \exp \left\{i t\left\|S_{n}-a\right\|^{2}\right\}\right)=\left(\begin{array}{l}
k \\
2
\end{array}\right) h_{1}(t), \\
H_{3}(t) & =\sum_{j=2}^{k-1} \sum_{i_{1}=1}^{j-1} \sum_{i_{2}=j+1}^{k} \mathbf{E}\left(\left(g_{1}\left(X_{j}\right), g_{2}\left(X_{i_{1}}, X_{i_{2}}\right)\right) \exp \left\{i t\left\|S_{n}-a\right\|^{2}\right\}\right)=\left(\begin{array}{l}
k \\
3
\end{array}\right) h_{2}(t), \\
H_{4}(t) & =\sum_{j=1}^{k-1} \sum_{i_{1}=j+1}^{k} \mathbf{E}\left(\left(g_{1}\left(X_{j}\right), g_{2}\left(X_{i_{1}}, X_{j}\right)\right) \exp \left\{i t\left\|S_{n}-a\right\|^{2}\right\}\right)=\left(\begin{array}{l}
k \\
2
\end{array}\right) h_{1}(t), \\
H_{5}(t)= & \sum_{j=1}^{k-2} \sum_{i_{2}=j+2}^{k} \sum_{i_{1}=j+1}^{i_{2}-1} \mathbf{E}\left(\left(g_{1}\left(X_{j}\right), g_{2}\left(X_{i_{1}}, X_{i_{2}}\right)\right) \exp \left\{i t\left\|S_{n}-a\right\|^{2}\right\}\right) \\
= & \left(\begin{array}{l}
k \\
3
\end{array}\right) h_{2}(t), \\
H_{6}(t)= & \sum_{i_{2}=2}^{k} \sum_{i_{1}=1}^{i_{2}-1} \mathbf{E}\left(\left(a, g_{2}\left(X_{i_{1}}, X_{i_{2}}\right)\right) \exp \left\{i t\left\|S_{n}-a\right\|^{2}\right\}\right)=\left(\begin{array}{l}
k \\
2
\end{array}\right) h_{3}(t) .
\end{aligned}
$$

These representations imply (32). Next we estimate functions $h_{i}(t)$ in (32). Obviously,

$$
\left\|g_{1 n}\left(X_{1}\right)+g_{1 n}\left(X_{2}\right)+S_{n 2}\right\|^{2}=\left\|S_{n 2}\right\|^{2}+W_{1}+W_{2},
$$


where

$$
W_{1}=\left\|g_{1 n}\left(X_{1}\right)+g_{1 n}\left(X_{2}\right)\right\|^{2}, \quad W_{2}=2\left(g_{1 n}\left(X_{1}\right)+g_{1 n}\left(X_{2}\right), S_{n 2}\right) .
$$

By (21) with $r=1$ and $z=\operatorname{it}\left(W_{1}+W_{2}\right)$, taking into consideration (2) we have

$$
\begin{aligned}
h_{1}(t)=i t \int_{0}^{1} d u \mathbf{E}( & \left(g_{1}\left(X_{1}\right), g_{2}\left(X_{1}, X_{2}\right)\right) W_{1} \exp \left\{i t u W_{1}\right\} \\
\times & \left.\mathbf{E}_{2} \exp \left\{i t\left\|S_{n 2}\right\|^{2}+i t u W_{2}\right\}\right) \\
+i t \int_{0}^{1} d u \mathbf{E}( & \left(g_{1}\left(X_{1}\right), g_{2}\left(X_{1}, X_{2}\right)\right) \exp \left\{i t u W_{1}\right\} \\
& \left.\times \mathbf{E}_{2}\left(\exp \left\{i t\left\|S_{n 2}\right\|^{2}+i t u W_{2}\right\} W_{2}\right)\right) .
\end{aligned}
$$

The expectation $\mathbf{E}_{2}$ with respect to $X_{3}, \ldots, X_{n}$ we will estimate using Lemma 3 . To estimate $h_{1}(t)$ in the interval (28) we take in Lemma $3 l \sim n / 2, m=n-l, A=1$. Then $T_{1}=|t|$ and

$$
\begin{aligned}
& \left|\mathbf{E}_{2} \exp \left\{i t\left\|S_{n 2}\right\|^{2}+i t u W_{2}\right\}\right| \\
& \left.\leqq c_{1} \quad \exp \left(-c_{2} n\right)+c_{3} L^{2} n^{-1}+\prod_{j=1}^{5}\left(1+c_{2} \sigma_{j}^{4} t^{2}\right)^{-1 / 4}\right) \\
& \left|\mathbf{E}_{2}\left(\exp \left\{i t\left\|S_{n 2}\right\|^{2}+i t u W_{2}\right\} W_{2}\right)\right| \leqq c_{1}(1+\|a\|)\left\|g_{1 n}\left(X_{1}\right)+g_{1 n}\left(X_{2}\right)\right\| \\
& \left.\quad \times \quad \exp \left(-c_{2} n\right)+c_{3} L^{2} n^{-1}+\prod_{j=1}^{5}\left(1+c_{2} \sigma_{j}^{4} t^{2}\right)^{-1 / 4}\right) .
\end{aligned}
$$

Moreover,

$$
\begin{aligned}
& \mathbf{E}\left(\left|\left(g_{1}\left(X_{1}\right), g_{2}\left(X_{1}, X_{2}\right)\right)\right|\left\|g_{1 n}\left(X_{1}\right)+g_{1 n}\left(X_{2}\right)\right\|\right) \\
& \quad \leqq n^{-1 / 2}\left(\mathbf{E}\left(\left\|g_{1}\left(X_{1}\right)\right\|^{2}\left\|g_{2}\left(X_{1}, X_{2}\right)\right\|\right)+\mathbf{E}\left(\left\|g_{1}\left(X_{1}\right)\right\|\left\|g_{1}\left(X_{2}\right)\right\|\left\|g_{2}\left(X_{1}, X_{2}\right)\right\|\right)\right) \\
& \quad \leqq 2 n^{-1 / 2}\left(\mathbf{E}\left\|g_{1}\right\|^{3}\right)^{2 / 3}\left(\mathbf{E}\left\|g_{2}\right\|^{3}\right)^{1 / 3}, \\
& \mathbf{E}\left(\left|\left(g_{1}\left(X_{1}\right), g_{2}\left(X_{1}, X_{2}\right)\right)\right|\left\|g_{1 n}\left(X_{1}\right)+g_{1 n}\left(X_{2}\right)\right\|^{2}\right) \\
& \quad \leqq 2 n^{-1 / 2}\left(\mathbf{E}\left\|g_{1}\right\|^{3}\right)^{2 / 3}\left(\mathbf{E}\left\|g_{2}\right\|^{3}\right)^{1 / 3} .
\end{aligned}
$$

Thus, by (33) and (34), in the interval (28)

$$
\begin{aligned}
\left|h_{1}(t)\right| \leqq & c_{1}(1+\|a\|)\left(\mathbf{E}\left\|g_{1}\right\|^{3}\right)^{2 / 3}\left(\mathbf{E}\left\|g_{2}\right\|^{3}\right)^{1 / 3} \\
& \left.\times \quad L n^{-1 / 2}+|t| \prod_{j=1}^{7}\left(1+c_{2} \sigma_{j}^{4} t^{2}\right)^{-1 / 4}\right) n^{-1 / 2} .
\end{aligned}
$$


To estimate $h_{1}(t)$ for $t$ satisfying (29) we take in Lemma $3, l \sim n / \log n, m=n-l$, $A={ }_{4}^{5}$. Then $T_{1}=T(m)$ and similar reasoning gives for $t$ satisfying (29)

$$
\begin{aligned}
\left|h_{1}(t)\right| \leqq & c_{1}|t| \\
& \times \quad(1+\|a\|)\left(\mathbf{E}\left\|g_{1}\right\|^{3}\right)^{2 / 3}\left(\mathbf{E}\left\|g_{2}\right\|^{3}\right)^{1 / 3} \\
& \quad \exp \left(-\frac{c_{2} n}{\log n}\right)+c_{3} L^{5 / 2} n^{-5 / 4}(\log n)^{5 / 4} \\
& \left.\quad+\prod_{j=1}^{5}\left(1+\frac{c_{4} \sigma_{j}^{4} n}{L^{2}(\log n) \log \left(m / L^{2}\right)}\right)^{-1 / 4}\right) n^{-1 / 2} \\
\leqq & c_{5}(1+\|a\|)\left(\mathbf{E}\left\|g_{1}\right\|^{3}\right)^{2 / 3}\left(\mathbf{E}\left\|g_{2}\right\|^{3}\right)^{1 / 3} c_{5}(V) L^{3 / 2} n^{-1} .
\end{aligned}
$$

Consider now $h_{2}(t)$ in (32). Denote

$$
\xi=\sum_{j=1}^{3} \xi_{j}, \quad \bar{\xi}=\sum_{j=1}^{3} \mathbf{E} g_{1 n}\left(X_{j}\right), \quad \xi_{j}=g_{1 n}\left(X_{j}\right)-\mathbf{E} g_{1 n}\left(X_{j}\right), j=1,2,3 .
$$

Obviously,

$$
\begin{aligned}
& \left\|g_{1 n}\left(X_{1}\right)+g_{1 n}\left(X_{2}\right)+g_{1 n}\left(X_{3}\right)+S_{n 3}\right\|=\left\|\xi+S_{n 3}+\bar{\xi}\right\|^{2} \\
& \quad=\left\|S_{n 3}+\bar{\xi}\right\|^{2}+W_{3}+W_{4}+W_{5},
\end{aligned}
$$

where

$$
W_{3}=\sum_{j=1}^{3}\left\|\xi_{j}\right\|^{2}, \quad W_{4}=2 \sum_{j=1}^{3}\left(\xi_{i}, S_{n 3}+\bar{\xi}\right), \quad W_{5}=2 \sum_{1 \leqq i<j \leqq 3}\left(\xi_{i}, \xi_{j}\right) .
$$

Applying (21) with $r=2$ and $z=i t W_{5}$ we can write

$$
h_{2}(t)=h_{21}(t)+h_{22}(t)+h_{23}(t),
$$

where

$$
\begin{aligned}
h_{21}(t)= & \left.\mathbf{E}\left(\left(g_{1}\left(X_{1}\right), g_{2}\left(X_{2}, X_{3}\right)\right) \exp \left\{i t\left\|S_{n 3}+\bar{\xi}\right\|^{2}+i t\left(W_{3}+W_{4}\right)\right)\right\}\right), \\
h_{22}(t)= & i t \mathbf{E}\left(\left(g_{1}\left(X_{1}\right), g_{2}\left(X_{2}, X_{3}\right)\right) W_{5} \exp \left\{i t\left\|S_{n 3}+\bar{\xi}\right\|^{2}+i t\left(W_{3}+W_{4}\right)\right\}\right), \\
h_{23}(t)= & -t^{2} \int_{0}^{1} d u(1-u) \mathbf{E}\left(\left(g_{1}\left(X_{1}\right), g_{2}\left(X_{2}, X_{3}\right)\right) W_{5}^{2}\right. \\
& \left.\times \exp \left\{i t\left\|S_{n 3}+\bar{\xi}\right\|^{2}+i t\left(W_{3}+W_{4}+u W_{5}\right)\right\}\right) .
\end{aligned}
$$

Furthermore, observe that

$$
\left|h_{23}(t)\right| \leqq t^{2} \mathbf{E}\left(\left|\left(g_{1}\left(X_{1}\right), g_{2}\left(X_{2}, X_{3}\right)\right)\right| W_{5}^{2}\left|\mathbf{E}_{3} \exp \left\{i t\left\|S_{n 3}+\bar{\xi}\right\|^{2}+i t W_{4}\right\}\right|\right) .
$$

In the interval (28) by Lemma 3 with $l \sim n / 2, m=n-l, A={ }_{2}^{3}$

$$
\begin{aligned}
& \left|\mathbf{E}_{3} \exp \left\{i t\left\|S_{n 3}+\bar{\xi}\right\|^{2}+i t W_{4}\right\}\right| \\
& \left.\quad \leqq c_{1} \quad \exp \left(-c_{2} n\right)+c_{3} L^{3} n^{-3 / 2}+\prod_{j=1}^{7}\left(1+c_{4} \sigma_{j}^{4} t^{2}\right)^{-1 / 4}\right),
\end{aligned}
$$


and for $t$ satisfying (29) Lemma 3 with $l \sim n / \log n, m=n-l, A={ }_{4}^{7}$ gives

$$
\begin{aligned}
&\left|\mathbf{E}_{3} \exp \left\{i t\left\|S_{n 3}+\bar{\xi}\right\|^{2}+i t W_{4}\right\}\right| \\
& \leqq c_{1} \quad \exp \left(-\frac{c_{2} n}{\log n}\right)+c_{3} L^{7 / 2} n^{-7 / 4}(\log n)^{7 / 4} \\
&\left.\quad+\prod_{j=1}^{7}\left(1+\frac{c_{4} \sigma_{j}^{4} n}{L^{2}(\log n) \log \left(m / L^{2}\right)}\right)^{-1 / 4}\right) .
\end{aligned}
$$

Moreover,

$$
\mathbf{E}\left(\left|\left(g_{1}\left(X_{1}\right), g_{2}\left(X_{2}, X_{3}\right)\right)\right| W_{5}^{2}\right) \leqq c\left(\mathbf{E}\left\|g_{1}\left(X_{1}\right)\right\|^{3}\right)^{2 / 3}\left(\mathbf{E}\left\|g_{2}\right\|^{3}\right)^{1 / 3} n^{-3 / 2}
$$

Combining (38)-(40) we obtain an estimate for $h_{23}(t)$.

By (21) with $r=1, z=i t\left(W_{3}+W_{4}\right)$ we have

$$
h_{22}(t)=F_{1}(t)+F_{2}(t),
$$

where

$$
\begin{gathered}
F_{1}(t)=i t \mathbf{E}\left(\left(\mathbf{E} g_{1}\left(X_{1}\right), g_{2}\left(X_{2}, X_{3}\right)\right) W_{5} \exp \left\{i t\left\|S_{n 3}+\bar{\xi}\right\|^{2}+i t\left(W_{3}+W_{4}\right)\right\}\right), \\
F_{2}(t)=-t^{2} \int_{0}^{1} d u \mathbf{E}\left(\left(g_{1}\left(X_{1}\right)-\mathbf{E} g_{1}\left(X_{1}\right), g_{2}\left(X_{2}, X_{3}\right)\right) W_{5}\left(W_{3}+W_{4}\right)\right. \\
\left.\times \exp \left\{i t\left\|S_{n 3}+\bar{\xi}\right\|^{2}+i t u\left(W_{3}+W_{4}\right)\right\}\right) .
\end{gathered}
$$

Here

$$
\left|F_{1}(t)\right| \leqq|t| \mathbf{E}\left(\left|\left(\mathbf{E} g_{1}\left(X_{1}\right), g_{2}\left(X_{2}, X_{3}\right)\right) W_{5}\right|\left|\mathbf{E}_{3} \exp \left\{i t\left\|S_{n 3}+\bar{\xi}\right\|^{2}+i t W_{4}\right\}\right|\right),
$$

and

$$
\mathbf{E}\left(\left|\left(\mathbf{E} g_{1}\left(X_{1}\right), g_{2}\left(X_{2}, X_{3}\right)\right)\right| W_{5}\right) \leqq 24\left(\mathbf{E}\left\|g_{2}\right\|^{2}\right)^{1 / 2} n^{-3 / 2} .
$$

Thus for $t$ satisfying (28) by Lemma 3 with $l \sim n / 2, m=n-l, A=1$

$$
\begin{aligned}
\left|F_{1}(t)\right| \leqq & c_{1}|t|\left(\mathbf{E}\left\|g_{2}\right\|^{2}\right)^{1 / 2} \\
& \left.\times c_{1} \quad \exp \left(-c_{2} n\right)+c_{3} L^{2} n^{-1}+\prod_{j=1}^{5}\left(1+c_{4} \sigma_{j}^{4} t^{2}\right)^{-1 / 4}\right) n^{-3 / 2},
\end{aligned}
$$

and for $t$ satisfying (29) by Lemma with $l \sim n / \log n, m=n-l$ and $A={ }_{4}^{5}$,

$$
\begin{aligned}
\left|F_{1}(t)\right| \leqq c_{1}|t|\left(\mathbf{E}\left\|g_{2}\right\|^{2}\right)^{1 / 2} c_{1}( & \exp \left(-\frac{c_{2} n}{\log n}\right)+c_{3} L^{5 / 2} n^{-5 / 4}(\log n)^{5 / 4} \\
& \left.+\prod_{j=1}^{5}\left(1+\frac{c_{4} \sigma_{j}^{4} n}{L^{2}(\log n) \log \left(m / L^{2}\right)}\right)^{-1 / 4}\right) n^{-3 / 2}
\end{aligned}
$$


To estimate $f_{2}(t)$ in (41) we first observe, that

$$
\begin{aligned}
\left|F_{2}(t)\right| \leqq t^{2} \int_{0}^{1} d u \mathbf{E}( & ||\left(g_{1}\left(X_{1}\right)-\mathbf{E} g_{1}\left(X_{1}\right), g_{2}\left(X_{2}, X_{3}\right)\right) W_{5} \mid \\
& \left.\times\left|\mathbf{E}_{3}\left(W_{3}+W_{4}\right) \exp \left\{i t\left\|S_{n 3}+\bar{\xi}\right\|^{2}+i t u W_{4}\right\}\right|\right),
\end{aligned}
$$

and

$$
\begin{aligned}
& \mathbf{E}\left(\left|\left(g_{1}\left(X_{1}\right)-\mathbf{E} g_{1}\left(X_{1}\right), g_{2}\left(X_{2}, X_{3}\right)\right) W_{5}\right|\left[W_{3}+\sum_{j=1}^{3}\left\|\xi_{j}\right\|(1+\|a\|)\right]\right) \\
& \quad \leqq c \mathbf{E}\left\|g_{1}\right\|^{3}\left(\mathbf{E}\left\|g_{2}\right\|^{3}\right)^{1 / 3}(1+\|a\|) n^{-3 / 2} .
\end{aligned}
$$

Applying now Lemma 3 for $t$ satisfying (28) with $l \sim n \log n, m=n-l$ and $A={ }_{2}^{3}$, we have

$$
\begin{aligned}
\left|F_{2}(t)\right| \leqq & c_{1} t^{2}(1+\|a\|) \mathbf{E}\left\|g_{1}\right\|^{3}\left(\mathbf{E}\left\|g_{2}\right\|^{3}\right)^{1 / 3} \\
& \left.\times \quad \exp \left(-c_{2} n\right) c_{3} L^{3} n^{-3 / 2}+\prod_{j=1}^{7}\left(1+c_{4} \sigma_{j}^{4} t^{2}\right)^{-1 / 4}\right) n^{-3 / 2}
\end{aligned}
$$

and for $t$ satisfy (29), using Lemma 3 with $l \sim n \log n, m=n-l$ and $A={ }_{4}^{7}$, we have

$$
\begin{aligned}
\left|F_{2}(t)\right| \leqq & c_{1} t^{2}(1+\|a\|) \mathbf{E}\left\|g_{1}\right\|^{3}\left(\mathbf{E}\left\|g_{2}\right\|^{3}\right)^{1 / 3} \\
& \times \quad \exp \left(-\frac{c_{2} n}{\log n}\right)+c_{3} L^{7 / 2} n^{-7 / 4}(\log n)^{7 / 4} \\
& \left.+\prod_{j=1}^{7}\left(1+\frac{c_{4} \sigma_{j}^{4} n}{L^{2}(\log n) \log \left(m / L^{2}\right)}\right)^{-1 / 4}\right) n^{-3 / 2}
\end{aligned}
$$

In (37) it remains only to estimate $h_{21}(t)$. We use the representation

$$
h_{21}(t)=F_{3}(t)+F_{4}(t),
$$

where

$$
\begin{aligned}
F_{3}(t)=\mathbf{E}( & \left.\left(\mathbf{E} g_{1}\left(X_{1}\right), g_{2}\left(X_{2}, X_{3}\right)\right) \exp \left\{i t\left\|S_{n 3}+\bar{\xi}\right\|^{2}+i t\left(W_{3}+W_{4}\right)\right\}\right), \\
F_{4}(t)=\mathbf{E}( & \left(g_{1}\left(X_{1}\right)-\mathbf{E} g_{1}\left(X_{1}\right), g_{2}\left(X_{2}, X_{3}\right)\right) \\
& \left.\times W_{5} \exp \left\{i t\left\|S_{n 3}+\bar{\xi}\right\|^{2}+i t\left(W_{3}+W_{4}\right)\right\}\right) .
\end{aligned}
$$

In view of (2) we can also write

$$
\begin{array}{ll}
F_{3}(t)=\mathbf{E} \quad\left(\mathbf{E} g_{1}\left(X_{1}\right), g_{2}\left(X_{2}, X_{3}\right)\right) \exp \left\{i t\left\|S_{n 3}+\bar{\xi}\right\|^{2}+i t\left\|\xi_{1}\right\|^{2}+2 i t\left(\xi_{1}, S_{n 3}+\bar{\xi}\right)\right\} \\
\left.\quad \times \prod_{j=2}^{3}\left(\exp \left\{i t\left\|\xi_{j}\right\|^{2}+2 i t\left(\xi_{j}, S_{n 3}+\bar{\xi}\right)\right\}-1\right)\right),
\end{array}
$$


$F_{4}(t)=\mathbf{E}\left(g_{1}\left(X_{1}\right)-\mathbf{E} g_{1}\left(X_{1}\right), g_{2}\left(X_{2}, X_{3}\right)\right) \exp \left\{i t\left\|S_{n 3}+\bar{\xi}\right\|^{2}\right\}$

$$
\left.\times \prod_{j=1}^{3}\left(\exp \left\{i t\left\|\xi_{j}\right\|^{2}+2 i t\left(\xi_{j}, S_{n 3}+\bar{\xi}\right)\right\}-1\right)\right) .
$$

Furthermore, applying (21) with $r=1$ in (47) and (48), we have

$$
\begin{aligned}
F_{3}(t)=-t^{2} \mathbf{E} \quad & \left(\mathbf{E} g_{1}\left(X_{1}\right), g_{2}\left(X_{2}, X_{3}\right)\right) \\
& \times \exp \left\{i t\left\|S_{n 3}+\bar{\xi}\right\|^{2}+i t\left\|\xi_{1}\right\|^{2}+2 i t\left(\xi_{1}, S_{n 3}+\bar{\xi}\right)\right\} \\
& \times \prod_{j=2}^{3}\left(\left\|\xi_{j}\right\|^{2}+2\left(\xi_{j}, S_{n 3}+\bar{\xi}\right)\right) \\
& \left.\times \int_{0}^{1} d u \exp \left\{i t u\left\|\xi_{j}\right\|^{2}+2 i t u\left(\xi_{j}, S_{n 3}+\bar{\xi}\right)\right\}\right), \\
F_{4}(t)=(i t)^{2} \mathbf{E} \quad & \left.g_{1}\left(X_{1}\right)-\mathbf{E} g_{1}\left(X_{1}\right), g_{2}\left(X_{2}, X_{3}\right)\right) \exp \left\{i t\left\|S_{n 3}+\bar{\xi}\right\|^{2}\right\} \\
& \times \prod_{j=1}^{3}\left(\left\|\xi_{j}\right\|^{2}+2\left(\xi_{j}, S_{n 3}+\bar{\xi}\right)\right) \\
& \left.\times \int_{0}^{1} d u \exp \left\{i t u\left\|\xi_{j}\right\|^{2}+2 i t u\left(\xi_{j}, S_{n 3}+\bar{\xi}\right)\right\}\right) .
\end{aligned}
$$

$$
b_{j}=\left\|\xi_{j}\right\|^{2}+2\left(\xi_{j}, \bar{\xi}\right)-2\left(\xi_{j}, a\right)
$$

Then

$$
\begin{gathered}
\prod_{j=2}^{3}\left(\left\|\xi_{j}\right\|^{2}+2\left(\xi_{j}, S_{n 3}+\bar{\xi}\right)\right)=\prod_{j=2}^{3}\left(b_{j}+2\left(\xi_{j}, S_{n 3}+a\right)\right) \\
(51) \quad=b_{2} b_{3}+2 b_{2}\left(\xi_{3}, S_{n 3}+a\right)+2 b_{3}\left(\xi_{2}, S_{n 3}+a\right)+4\left(\xi_{2}, S_{n 3}+a\right)\left(\xi_{3}, S_{n 3}+a\right),
\end{gathered}
$$

where according to the notation introduced above

$$
S_{n 3}+a=n^{-1 / 2}\left(g_{1}\left(X_{4}\right)+\cdots+g_{1}\left(X_{n}\right)\right) .
$$

Note that

$$
\begin{aligned}
&\left\|\xi_{j}\right\| \leqq 2, \quad\left\|\xi_{j}\right\| \leqq n^{-1 / 2}\left(1+\left\|g_{1}\left(X_{j}\right)\right\|\right), \\
&\left|b_{j}\right| \leqq 4(1+\|a\|)\left(1+\left\|g_{1}\left(X_{j}\right)\right\|\right) n^{-1 / 2}
\end{aligned}
$$

Substituting now (51) in (49) we obtain four expectations. Then we apply Lemma 3 to each of them, taking into account (52) and the estimate $\left\|\mathbf{E} g_{1}\left(X_{1}\right)\right\| \leqq n^{-1 / 2}$. For $t$ satisfying (28) we take in Lemma $3 l \sim n / 2, m=n-l, A={ }_{2}^{3}$ and obtain

$$
\left|F_{3}(t)\right| \leqq c_{1} t^{2}(1+\|a\|)^{2}\left(\mathbf{E}\left\|g_{2}\right\|^{2}\right)^{1 / 2}
$$




$$
\left.\times \quad \exp \left(-c_{2} n\right)+c_{3} L^{3} n^{-3 / 2}+\prod_{j=1}^{7}\left(1+c_{4} \sigma_{j}^{4} t^{2}\right)^{-1 / 4}\right) n^{-3 / 2},
$$

and for $t$ satisfying (29) we take $l \sim n / \log n, m=n-l$ and $A={ }_{4}^{7}$ and obtain

$$
\begin{aligned}
\left|F_{3}(t)\right| \leqq & c_{1} t^{2}(1+\|a\|)^{2}\left(\mathbf{E}\left\|g_{2}\right\|^{2}\right)^{1 / 2} \\
& \times \quad \exp \left(-\frac{c_{2} n}{\log n}\right)+c_{3} L^{7 / 2} n^{-7 / 4}(\log n)^{7 / 4} \\
& \left.+\prod_{j=1}^{7}\left(1+\frac{c_{4} \sigma_{j}^{4} n}{L^{2}(\log n) \log \left(n / L^{2}\right)}\right)^{-1 / 4}\right) n^{-3 / 2} .
\end{aligned}
$$

To estimate $f_{4}(t)$ in $(50)$ we use the following formula which is analogous to (51):

$$
\begin{aligned}
& \prod_{j=1}^{3}\left(\left\|\xi_{j}\right\|^{2}+2\left(\xi_{j}, S_{n 3}+\bar{\xi}\right)\right)=b_{1} b_{2} b_{3}+2 b_{1} b_{2}\left(\xi_{3}, S_{n 3}+a\right) \\
& \quad+2 b_{1} b_{3}\left(\xi_{2}, S_{n 3}+a\right)+2 b_{2} b_{3}\left(\xi_{1}, S_{n 3}+a\right)+4 b_{1}\left(\xi_{2}, S_{n 3}+a\right)\left(\xi_{3}, S_{n 3}+a\right) \\
& \quad+4 b_{2}\left(\xi_{1}, S_{n 3}+a\right)\left(\xi_{3}, S_{n 3}+a\right)+4 b_{3}\left(\xi_{1}, S_{n 3}+a\right)\left(\xi_{2}, S_{n 3}+a\right) \\
& \quad+8\left(\xi_{1}, S_{n 3}+a\right)\left(\xi_{2}, S_{n 3}+a\right)\left(\xi_{3}, S_{n 3}+a\right) .
\end{aligned}
$$

Substituting it in (50) we obtain eight similar expectations. To estimate these expectations we again apply Lemma 3. For $t$ satisfying (28) we take in it $l \sim n / 2, m=n-l$, $A=2$ and obtain,

$$
\begin{aligned}
\left|F_{4}(t)\right| \leqq & c_{1}|t|^{3}(1+\|a\|)^{3}\left(\mathbf{E}\left\|g_{2}\right\|^{2}\right)^{1 / 2} \\
& \left.\times \exp \left(-c_{2} n\right)+c_{3} L^{4} n^{-2}+\prod_{j=1}^{9}\left(1+c_{4} \sigma_{j}^{4} t^{2}\right)^{-1 / 4}\right) n^{-3 / 2},
\end{aligned}
$$

and for $t$ satisfying (29) we take $l \sim n / \log n, m=n-l$ and $A={ }_{4}^{9}$ and obtain

$$
\begin{aligned}
\left|F_{4}(t)\right| \leqq & c_{1}|t|^{3}(1+\|a\|)^{3}\left(\mathbf{E}\left\|g_{2}\right\|^{2}\right)^{1 / 2} \\
& \times \quad \exp \left(-\frac{c_{2} n}{\log n}\right)+c_{3} L^{9 / 2} n^{-9 / 4}(\log n)^{9 / 4} \\
& \left.\quad+\prod_{j=1}^{9}\left(1+\frac{c_{4} \sigma_{j}^{4} n}{L^{2}(\log n) \log \left(m / L^{2}\right)}\right)^{-1 / 4}\right) n^{-3 / 2}
\end{aligned}
$$

Estimates (37)-(38), (41)-(42), (53), and (55) together for $t$ satisfying (28) imply

$$
\begin{aligned}
\left|h_{2}(t)\right| \leqq & c_{1}(1+\|a\|)^{3}\left(\mathbf{E}\left\|g_{1}\right\|^{3}\right)\left(\mathbf{E}\left\|g_{2}\right\|^{3}\right)^{1 / 3} \\
& \times \quad n^{-1 / 2}+\left(|t|+t^{2}\right) \prod_{j=1}^{7}\left(1+c_{4} \sigma_{j}^{4} t^{2}\right)^{-1 / 4} \\
& \left.\quad+|t|^{3} \prod_{j=1}^{9}\left(1+c_{4} \sigma_{j}^{4} t^{2}\right)^{-1 / 4}\right) n^{-3 / 2}
\end{aligned}
$$


and (37), (39), (41), (43), (54), and (56) for $t$ satisfying (29) imply

$$
\left|h_{2}(t)\right| \leqq c_{1}(1+\|a\|)^{3}\left(\mathbf{E}\left\|g_{1}\right\|^{3}\right)\left(\mathbf{E}\left\|g_{2}\right\|^{3}\right)^{1 / 3} c_{9}(V) L^{3 / 2} n^{-2} .
$$

It remains to estimate $h_{3}(t)$ in (32). Applying (21) with $r=1$ and $z=i t\left(W_{1}+\right.$ $\left.W_{2}\right), z=i t u\left(W_{1}+W_{2}\right)$ twice, we have

$$
\begin{aligned}
h_{3}(t)=i t \int_{0}^{1} d u \mathbf{E}\left(\left(a, g_{2}\left(X_{1}, X_{2}\right)\right) W_{1} \exp \left\{i t u W_{1}\right\} \mathbf{E}_{2} \exp \left\{i t\left\|S_{n 2}\right\|^{2}+i t u W_{2}\right\}\right) \\
-t^{2} \int_{0}^{1} d v \int_{0}^{1} d u u \mathbf{E}\left(\left(a, g_{2}\left(X_{1}, X_{2}\right)\right) \exp \left\{i t u v W_{1}\right\}\right. \\
\left.\times \mathbf{E}_{2}\left(\exp \left\{i t\left\|S_{n 2}\right\|^{2}+i t u v W_{2}\right\}\left(W_{1}+W_{2}\right)\right)\right) .
\end{aligned}
$$

Arguing in the same way as in proving (35) and (36) we obtain for $t$ satisfying (28)

$$
\begin{aligned}
\left|h_{3}(t)\right| \leqq & c_{1}(1+\|a\|)^{3}\left(\mathbf{E}\left\|g_{1}\right\|^{3}\right)^{2 / 3}\left(\mathbf{E}\left\|g_{2}\right\|^{3}\right)^{1 / 3} \\
& \times\left[L n^{-1 / 2}+\left(|t|+t^{2}\right) \prod_{j=1}^{7}\left(1+c_{2} \sigma_{j}^{4} t^{2}\right)^{-1 / 4}\right] n^{-1},
\end{aligned}
$$

and for $t$ satisfying (29)

$$
\left|h_{3}(t)\right| \leqq c_{1}\left(1+\|a\|^{3}\right)\left(\mathbf{E}\left\|g_{1}\right\|^{3}\right)^{2 / 3}\left(\mathbf{E}\left\|g_{2}\right\|^{3}\right)^{1 / 3} c_{7}(V) L^{3 / 2} n^{-3 / 2} .
$$

Now (32), (35), (57), (59) imply (30) and (32), (36), (58), (60) imply (31).

Lemma 6. If $t$ belongs to the interval (28), then

$$
\begin{aligned}
\left|J_{2}(t)\right| \leqq & c_{1}\left(1+\|a\|^{2}\right)\left(\mathbf{E}\left\|g_{1}\right\|^{3}\right)^{2 / 3} \\
& \left.\times L^{2} n^{-1 / 2}+n^{1 / 6} \prod_{j=1}^{9}\left(1+\frac{c_{2} \sigma_{j}^{4} t^{2}}{n^{1 / 3}}\right)^{-1 / 6}\right) n^{-1},
\end{aligned}
$$

and if $t$ satisfies (29)

$$
\left|J_{2}(t)\right| \leqq c_{1}\left(1+\|a\|^{2}\right)\left(\mathbf{E}\left\|g_{1}\right\|^{3}\right)^{2 / 3} c_{9}(V) L^{3} n^{-3 / 2} .
$$

Proof. Noting that

$$
J_{2}(t)=\mathbf{E}\left(Z_{n}^{2} \mathbf{E} \exp \left\{i t\left\|S_{n}-a\right\|^{2}\right\}\right)
$$

we have

$$
\begin{aligned}
\left|J_{2}(t)\right| \leqq & 4 \varepsilon_{n}^{2} \mathbf{E}\left(\left\|S_{n}^{k}\right\|^{2}\left\|\alpha_{k}\right\|^{2}\left|\mathbf{E}_{k} \exp \left\{i t\left\|S_{n}-a\right\|^{2}\right\}\right|\right) \\
& +4 \varepsilon_{n}^{2}\|a\|^{2} \mathbf{E}\left(\left\|\alpha_{k}\right\|^{2}\left|\mathbf{E}_{k} \exp \left\{i t\left\|S_{n}-a\right\|^{2}\right\}\right|\right) .
\end{aligned}
$$

By Hölder's inequality

$$
\begin{aligned}
& \mathbf{E}\left(\left\|S_{n}^{k}\right\|^{2}\left\|\alpha_{k}\right\|^{2}\left|\mathbf{E}_{k} \exp \left\{i t\left\|S_{n}-a\right\|^{2}\right\}\right|\right) \\
& \quad \leqq\left(\mathbf{E}\left\|\alpha_{k}\right\|^{3}\right)^{2 / 3}\left(\mathbf{E}\left(\left\|S_{n}^{k}\right\|^{6}\left|\mathbf{E}_{k} \exp \left\{i t\left\|S_{n}-a\right\|^{2}\right\}\right|^{3}\right)\right)^{1 / 3} .
\end{aligned}
$$


Since for any $x>0$ and $p>0$

$$
\left\|S_{n}^{k}\right\|^{6} \leqq x^{6}+x^{-p}\left\|S_{n}^{k}\right\|^{p+6}
$$

we have in (64)

$$
\begin{aligned}
& \mathbf{E}\left(\left\|S_{n}^{k}\right\|^{6}\left|\mathbf{E}_{k} \exp \left\{i t\left\|S_{n}-a\right\|^{2}\right\}\right|^{3}\right) \\
& \quad \leqq x^{6} \mathbf{E}\left|\mathbf{E}_{k} \exp \left\{i t\left\|S_{n}-a\right\|^{2}\right\}\right|^{2}+x^{-p} \mathbf{E}\left\|S_{n}^{k}\right\|^{p+6} .
\end{aligned}
$$

To estimate the second expectation in (63) we also use Hölder's inequality and obtain

$$
\begin{aligned}
\mathbf{E}\left(\left\|\alpha_{k}\right\|^{2}\left|\mathbf{E}_{k} \exp \left\{i t\left\|S_{n}-a\right\|^{2}\right\}\right|\right) & \leqq \mathbf{E}\left(\left\|\alpha_{k}\right\|^{3}\right)^{2 / 3}\left(\mathbf{E}\left|\mathbf{E}_{k} \exp \left\{i t\left\|S_{n}-a\right\|^{2}\right\}\right|^{3}\right)^{1 / 3} \\
& \leqq \mathbf{E}\left(\left\|\alpha_{k}\right\|^{3}\right)^{2 / 3}\left(\mathbf{E}\left|\mathbf{E}_{k} \exp \left\{i t\left\|S_{n}-a\right\|^{2}\right\}\right|^{2}\right)^{1 / 3} .
\end{aligned}
$$

Substituting (64) in (63) and using the elementary inequality $(\alpha+\beta)^{1 / 3} \leqq \alpha^{1 / 3}+\beta^{1 / 3}$ we get

$$
\begin{aligned}
\left|J_{2}(t)\right| \leqq & 4 \varepsilon_{n}^{2} \mathbf{E}\left(\left\|\alpha_{k}\right\|^{3}\right)^{2 / 3} \\
& \times\left[x^{-p / 3}\left(\mathbf{E}\left\|S_{n}^{k}\right\|^{p+6}\right)^{1 / 3}+\left(x^{2}+\|a\|^{2}\right)\left(\mathbf{E} \mid \mathbf{E}_{k} \exp \left\{\left.i t\left\|S_{n}-a\right\|^{2}\right|^{2}\right)^{1 / 3}\right]\right.
\end{aligned}
$$

Applying the same argument as in the proof of Lemma 10 in [3] with $l \sim n^{2 / 3}$, $m=n-l, A={ }_{2}^{3}$ for $|t| \leqq c n^{1 / 2} L^{-1}$, we have

$$
\left.\mathbf{E}\left|\mathbf{E}_{k} \exp \left\{i t\left\|S_{n}-a\right\|\right\}\right|^{2} \leqq c_{1} L^{3} n^{-1}+\prod_{j=1}^{9}\left(1+\frac{c_{2} \sigma_{j}^{4} T_{1}^{2}}{n^{1 / 3}}\right)^{-1 / 2}\right),
$$

where $T_{1}$ is defined in Lemma 3. Furthermore, taking in (67) $p=36, x=n^{1 / 12}$ and using (8) and (9) we obtain the estimate

$$
\begin{aligned}
\left|J_{2}(t)\right|= & c_{1}\left(1+\|a\|^{2}\right)\left(\mathbf{E}\left\|g_{2}\right\|^{3}\right)^{2 / 3} \\
& \left.\times L^{2} n^{-1 / 2}+n^{1 / 6} \prod_{j=1}^{9}\left(1+\frac{c_{2} \sigma_{j}^{4} T_{1}^{2}}{n^{1 / 3}}\right)^{-1 / 6}\right) n^{-1},
\end{aligned}
$$

which implies (61) and (62).

LEMMA 7. $I_{2}$ in (19) satisfies the inequality

$$
I_{2} \leqq c_{1}\left(1+\|a\|^{3}\right) \beta^{2} \sigma_{9}^{-1} c_{9}(V) n^{-1 / 2} .
$$

Proof. (22) implies

$$
\left|\varphi_{n}(t)-\psi_{n}(t)\right||t|^{-1} \leqq\left|J_{1}(t)\right|+|t|\left|J_{2}(t)\right|+t^{2}\left|J_{3}(t)\right| .
$$

By Lemma 4

$$
\int_{-T}^{T} t^{2}\left|J_{3}(t)\right| d t \leqq c_{1}\left(1+\|a\|^{3}\right) \mathbf{E}\left\|g_{1}\right\|^{3} \mathbf{E}\left\|g_{2}\right\|^{3} c_{7}(V) n^{-1 / 2} .
$$


By Lemma 5

$$
\int_{-T}^{T}\left|J_{1}(t)\right| d t \leqq c_{1}\left(1+\|a\|^{3}\right)\left(\mathbf{E}\left\|g_{1}\right\|^{3}\right)\left(\mathbf{E}\left\|g_{2}\right\|^{3}\right)^{1 / 3} c_{9}(V) L^{1 / 2} n^{-1 / 2} .
$$

By Lemma 6

$$
\int_{-T}^{T}|t|\left|J_{2}(t)\right| d t \leqq c_{1}\left(1+\|a\|^{2}\right)\left(\mathbf{E}\left\|g_{1}\right\|^{3}\right)^{2 / 3} c_{9}(V) L n^{-1 / 2} .
$$

Noting now that the moments in (70)-(72) satisfy (4) and integrating both sides in (69), we obtain (68).

From (14), (19)-(20), and (68) it follows that

$$
\Delta_{n 2}(a) \leqq c_{1}\left(1+\|a\|^{3}\right) \beta^{2} \sigma_{9}^{-1} c_{9}(V) n^{-1 / 2} .
$$

Estimates (5), (6), and (73) imply (3) and the theorem is proved.

\section{REFERENCES}

[1] Yu. V. Borovskikh, Theory of U-statistics in Hilbert space, Preprint No 86.78, Institute of Mathematics, Ukraine Academy of Science, Kiev, 1986 (in Russian).

[2] Yu. V. Borovskikh And V. S. Korolyuk, UH-statistics, Soviet Math. Dokl., 40 (1990), pp. 432435.

[3] B. A. Zalesskit, V. V. Sazonov, and V. V. Ul'yanov, Normal approximation in Hilbert space, I, II, Theory Probab. Appl., 33 (1988), pp. 207-227; pp. 473-487.

[4] V. S. Koroljuk and Yu. V. Borovskikh, Approximation of nondegenerate U-statistics, Theory Probab. Appl., 30 (1986), pp. 439-450.

[5] V. S. Korolyuk and Yu. V. Borovskikh, Theory of U-statistics, Kluwer, Dordrecht, 1994.

[6] V. Paulauskas and A. RaČKauskas, Approximation Theory in the Central Limit Theorem. Exact Results in Banach Spaces, Kluwer, Dordrecht, 1989.

[7] V. V. Petrov, Sums of Independent Random Variables, Springer-Verlag, Berlin, 1975.

[8] I. F. Pinelis, Estimates of moments of infinite dimensional martingales, Math. Notes, 27 (1980), pp. 459-462.

[9] B. A. Zalesskit, V. V. Sazonov, and V. V. Ul'yanov, A precise estimate of the rate of convergence in the central limit theorem in Hilbert space, Math. USSR Sbornik, 68 (1991), pp. $453-482$.

[10] V. Bentkus, F. Götze, And R. Zitikis, Lower estimates of the convergence rate for U-statistics, Ann. Probab., 22 (1994), No. 4.

[11] W. S. Grams and R. J. Serfling, Convergence rate for U-statistics., Ann. Statist., 1 (1973), pp. 153-160.

[12] V. S. Korolyuk and Yu. V. Borovskikh, Rate of convergence in the central limit theorem for UH-statistics, Theory Probab. Math. Statist., 43 (1991), pp. 79-85.

[13] A. J. Lee, U-statistics; Theory and Practice, Marcel Dekker, New York, 1990.

[14] M. L. Puri And V. V. Sazonov, On Hilbert space valued U-statistics, Theory Probab. Appl., 36 (1991), pp. 604-605.

[15] V. V. Sazonov, Normal Approximation - Some Recent Advances, Lecture Notes in Math., Vol. 879, Springer-Verlag, Berlin, 1981.

[16] V. V. Sazonov, V. V. Ul'yanov, and B. A. Zalesskil, Asymptotically precise estimate of accuracy of Gaussian approximation in Hilbert space, J. Multivariate Anal., 28 (1989), pp. 304-330.

[17] V. V. Sazonov and V. V. Ul'yanov, Speed of convergence in the central limit theorem in Hilbert space under weakened moment conditions, in Proc. 5th Vilnius Conference on Probability Theory and Mathematical Statistics, 1989, Mokslas/VSP, Vilnius/Utrecht, 1990, pp. 394410. 